\title{
Effect of static and dynamic muscle stretching as part of warm up procedures on knee joint proprioception and strength.
}

Gregory S Walsh

Oxford Brookes University, Department of Sport and Health Sciences, Oxford, UK

Walsh, GS. (2017). Effect of static and dynamic muscle stretching as part of warm up procedures on knee joint proprioception and strength. Human Movement Science, 55, 189195. doi: https://doi.org/10.1016/j.humov.2017.08.014

\section{Abstract}

Background: The importance of warm up procedures prior to athletic performance is well established. A common component of such procedures is muscle stretching. There is conflicting evidence regarding the effect of static stretching (SS) as part of warm up procedures on knee joint position sense (KJPS) and the effect of dynamic stretching (DS) on KJPS is currently unknown. The aim of this study was to determine the effect of dynamic and static stretching as part warm up procedures on KJPS and knee extension and flexion strength.

Methods: This study had a randomised cross-over design and ten healthy adults $(20 \pm 1$ years) attended 3 visits during which baseline KJPS, at target angles of $20^{\circ}$ and $45^{\circ}$, and knee extension and flexion strength tests were followed by 15 minutes of cycling and either a rest period (CON), SS, or DS and repeat KJPS and strength tests. All participants performed all conditions, one condition per visit.

Results: There were warm up $x$ stretching type interactions for KJPS at $20^{\circ}(p=0.024)$ and $45^{\circ}(p=0.018)$, and knee flexion $(p=0.002)$ and extension $(p<0.001)$ strength. The SS and DS improved KJPS but CON condition did not and SS decreased strength. No change in strength was present for DS or CON. 
Conclusions: Both SS and DS improve KJPS as part of pre-exercise warm up procedures. However, the negative impact of SS on muscle strength limits the utility of SS before athletic performance. If stretching is to be performed as part of a warm up, DS should be favoured over SS.

Keywords: Proprioception; Warm-Up Exercise; Muscle Strength; Static Stretching; Dynamic Stretching; Joint Position Sense

\section{Introduction}

Proprioception is the sense of the state, position and velocity, of body segments relative to neighbouring segments. Improvements in proprioception have been associated with decreased injury risk (Mandelbaum et al., 2005; Ogard, 2011), and can be achieved following moderate intensity warm up exercise (Bartlett \& Warren, 2002; Bouët \& Gahéry, 2000; Magalhães, Ribeiro, Pinheiro, \& Oliveira, 2010; Subasi, Gelecek, \& Aksakoglu, 2008). Pre-exercise warm up procedures therefore can have an important contribution to reducing the immediate risk of soft tissue injury (Daneshjoo, Mokhtar, Rahnama, \& Yusof, 2012).

During pre-exercise warm up procedures moderate intensity exercise is often performed in conjunction with stretching exercises (Bishop, 2003). There is conflicting evidence regarding the effect of stretching on proprioception. Static stretching (SS) of shoulder adduction agonists and antagonists had no effect on shoulder joint position sense (JPS) (Björklund, Djupsjöbacka, \& Crenshaw, 2006). Furthermore, no change in knee JPS (KJPS) was found after SS of the knee extensors and flexors (Larsen et al., 2005). However, using the same duration of stretch, of the same muscle groups, Ghaffarinejad et al. (2007) reported improved KJPS. 
Studies examining the effect of stretching on proprioception have focussed on SS (Larsen et al., 2005; Björklund et al., 2006; Ghaffarinejad et al., 2007). In recent times the utility of SS as exercise preparation has been questioned (Simic, Sarabon, \& Markovic, 2013), because acute effects of SS include decreased muscle strength (Cramer et al., 2004), sprint performance ( Fletcher \& Anness, 2007; Haddad et al., 2014), and increased fatigability (Trajano, Pinho, Costa, \& Oliveira, 2015). An alternative of increasing popularity is dynamic stretching (DS), which involves a repeated controlled movement through the joint range of motion (Fletcher \& Anness, 2007). Dynamic stretching improves muscle strength (Sekir, Arabaci, Akova, \& Kadagan, 2010), sprint (Haddad et al., 2014) and jumping (Haddad et al., 2014; Ryan et al., 2014) performance. However, the effect of dynamic stretching as part of a warm up procedure on KJPS is currently unknown.

The aim of this study was to determine the effects of SS and DS, following warm up exercise, on KJPS, and knee extension and flexion muscle strength. It was hypothesised that SS and DS would improve KJPS when compared to performing warm up exercise only. In addition, it was hypothesised that SS would decrease strength and DS would have opposing effects, when compared to performing warm up exercise only.

\section{Methods}

\subsection{Participants}

Participants included 10 ( 3 females, 7 males, mean age $20 \pm 1$ years, mean mass $66.9 \pm$ $10.5 \mathrm{~kg}$, mean height: $169.6 \pm 8.9 \mathrm{~cm}$ ) healthy physically active adults. Student athletes competing in the sports of Football (soccer), Rugby Union and Tennis participated in the study. Participants had no current lower limb injury, or history of knee joint reconstruction. This study was approved by the University ethics committee. All participants gave written informed consent, were aware of the nature of the study, and their right to withdraw, before participating.

\subsection{Study Design}


The study had a randomised cross-over design consisting of 3 experimental conditions. Participants attended 3 laboratory visits during which a single experimental condition was performed. Each participant performed each condition, one per visit, and visits were separate by a period of 3-5 days. Each experimental condition consisted of a baseline (pre) measurement of KJPS, and knee concentric extension and flexion strength. At the beginning of each visit participants performed 3 practice trials of each of the KJPS conditions and knee extension and flexion isokinetic strength tests. Baseline measurements were followed by a period of 15 minutes cycling (Monark Exercise AB, Sweden) at $70 \%$ of the participant's estimated maximum heart rate. Participants then performed either seated rest (CON), SS, or DS stretching, immediately followed by post-test KJPS and knee extension and flexion strength measurements (Figure 1).

\subsection{Stretching Procedures}

Static stretches of the quadriceps and hamstrings muscle groups were held for 90 seconds each, held a point of mild discomfort. Dynamic stretches were performed for 3 sets of 12 repetitions of each movement at a self-preferred velocity, 30 seconds of rest was provided between each set. Each stretching protocol, and seated rest, lasted 3 minutes. For both SS and DS the order in which the muscles groups were stretched was counterbalanced between participants to avoid potential order effects.

Written and verbal instructions were provided for each stretch. For the quadriceps SS, participants stood upright and flexed the dominant leg, the ankle was then grasped and the knee flexed until the foot approached the buttocks, the hips were slightly extended to increase the stretch. For the hamstring SS, participants sat on the floor with the dominant leg extended and non-dominant leg flexed so the sole of the foot was against the inside of the thigh. Participants then leaned forward and grasped either leg or foot as far as they could reach and maintain for the duration of the stretch. For the quadriceps DS, participants took a large step forward with the non-dominant leg and flexed the knee with foot flat on the floor into a lunge position. The non-dominant knee was flexed while the dominant knee flexed 
with the hip in an extended position until the dominant knee touched the floor before returning to the standing position. For the hamstring DS, participants stood upright using one hand to maintain balance, while contracting the hip flexors of the dominant leg to bring the leg forward until a point of mild discomfort in the hamstrings was reached, before allowing the leg to return down to the start position. For all DS stretches, each stretch repetition was performed for the complete range of motion described. The degree of stretch in both SS and DS was determined as participant's self-reported end of range of motion for each movement.

\subsection{Knee Joint Position Sense Measurement}

Knee joint position sense was measured actively using an isokinetic dynamometer (Biodex Medical Systems, New York, USA) at an angle of $20^{\circ}$ and $45^{\circ}$ for the dominant limb, determined as the limb participants chose to kick a ball. The hip angle was set at $85^{\circ}$ and each KJPS measurement started with the knee flexed at $90^{\circ}$. The participants lower leg was moved at a speed of $10^{\circ} / \mathrm{s}$ to the target position (either $20^{\circ}$ or $45^{\circ}$ in the direction of extension) and held for 5 seconds before being returned to $90^{\circ}$ flexion. The knee joint was then actively extended at a speed of $10 \%$, before participants manually stopped the dynamometer motion when they perceived the knee to have reached the target angle. Participants were blindfolded for the duration of the KJPS test to avoid visual input.

Three repetitions were performed for both the $20^{\circ}$ and $45^{\circ} \mathrm{KJPS}$ tests at baseline and post experimental conditions. The order in which the KJPS conditions (target angle of $20^{\circ}$ or $45^{\circ}$ ) were performed was randomised for each visit, to prevent order effects. Knee joint position sense was calculated as the absolute error (AE) between the target angle and the actively recreated angle. The average of the 3 trials for $20^{\circ}$ and $45^{\circ}$ respectively at each stage was used for statistical analysis.

\subsection{Strength Measurement}

Concentric knee extension and flexion strength were measured using an isokinetic dynamometer in a seated position, with the hips fixed at an angle of $85^{\circ}$. Participants 
performed 3 sets of 5 repetitions of concentric knee extension and flexion contractions at a speed of $120 \%$ s. Sixty seconds of rest was provided between each set. Concentric flexion and extension peak torque were determined as the average of the maximum torque achieved during each set.

\subsection{Statistics}

Two separate two-way repeated measures MANOVAs were performed to determine the effects of warm up (pre, post) and stretching type (SS, DS, CON) on KJPS measured at $20^{\circ}$ and $45^{\circ}$, and on concentric quadriceps and hamstrings peak torque. Separate MANOVA tests were performed of KJPS and strength variables to avoid low intercorrelations between the KJPS and strength variables decreasing the power of the MANOVA and potentially masking independent effects for KJPS and strength. Wilk's lambda $(\lambda)$ test statistic was used for MANOVAs. Univariate tests were performed to explore significant multivariate effects, and pairwise comparisons with Bonferroni correction were performed for significant main effects. Effects sizes were estimated using partial eta squared $\left(\eta_{p}^{2}\right)$ and were interpreted as small: $0.10-0.29$, medium: $0.30-0.49$ and large: $\geq 0.50$ (Cohen, 1988). An alpha level of 0.05 was used for all statistical tests.

\section{Results}

\subsection{Effect of Warm Up and Stretching on KJPS}

There was a warm up $x$ stretching type multivariate interaction effect for KJPS at $20^{\circ}$ and $45^{\circ}\left(\lambda=2.93, F(4,34)=2.93, p=0.035, \eta_{p}^{2}=0.26\right)$ and main effect of warm up $(\lambda=0.19$, $\left.\mathrm{F}(2,8)=16.86, \mathrm{p}=0.001, \eta_{p}^{2}=0.81\right)$ on $\mathrm{KJPS}$ at $20^{\circ}$ and $45^{\circ}$. Univariate warm up $\mathrm{x}$ stretching type interactions were present for KJPS at $20^{\circ}\left(\mathrm{F}(2,18)=2.54, \mathrm{p}=0.024, \eta_{p}^{2}=\right.$ $0.25)$ and $45^{\circ}\left(\mathrm{F}(2,18)=2.87, \mathrm{p}=0.018, \eta_{p}^{2}=0.27\right)$. The KJPS AE at $20^{\circ}$ and $45^{\circ}$ was decreased after both DS and SS but unchanged after CON (Figure 1). The KJPS AE at $45^{\circ}$ was lower after warm up compared to pre $\left(F(1,9)=24.88, p=0.001, \eta_{p}^{2}=0.73\right)$.

\subsection{Effect of Warm Up and Stretching on Knee Extension and Flexion Strength}


There was a warm up $x$ stretching type multivariate interaction effect for knee extension and flexion strength $\left(\lambda=0.18, F(4,34)=11.42, p<0.001, \eta_{p}^{2}=0.57\right)$ and multivariate effect of stretching type $\left(\lambda=0.48, F(4,34)=0.48, \mathrm{p}=0.013, \eta_{p}^{2}=0.30\right)$ on knee extension and flexion strength. Univariate warm up $\mathrm{x}$ stretching type interaction effects were present for flexion $\left(\mathrm{F}(2,18)=8.88, \mathrm{p}=0.002, \eta_{p}^{2}=0.50\right)$ and extension $\left(\mathrm{F}(2,18)=38.45, \mathrm{p}<0.001, \eta_{p}^{2}=0.81\right)$ strength. Static stretching decreased strength for both muscle groups (Figure 2). There was a univariate effect of stretching type on knee extension strength $(F(2,18)=7.81, p=0.004$, $\left.\eta_{p}^{2}=0.47\right)$, but no effect on knee flexion strength. Knee extension strength was lower in SS than in DS $(p=0.025)$ and CON $(p=0.036)$ conditions.

\section{Discussion}

The main findings of this study were that interaction effects between warm up and stretching type exist for both KJPS and knee muscle strength. There was a main effect of warm up, and both SS and DS improved KJPS compared to CON. Furthermore, SS decreased extension and flexion concentric strength compared to CON and DS, in partial agreement with the hypothesised effect as CON and DS had no effect on muscle strength.

The findings of the present study appear to agree with those of a previous study (Ghaffarinejad et al., 2007). Knee joint position sense at a target angle of $45^{\circ}$ was improved by warm up, and the significant interaction suggests that performing either SS or DS in addition to a period of moderate intensity cycling improves KJPS compared to cycling alone. These findings contrast with those reporting the effect of static stretching on KJPS (Larsen et al., 2005) and shoulder JPS (Björklund et al., 2006), that report no effect of stretching. A potential explanation for this difference is that in the current study static stretching consisted of a 90 second continuous stretch as opposed to 330 second periods of stretching (Larsen et al., 2005), or 320 second periods of stretching, adopted previously (Björklund et al., 2006). It is possible that the shorter periods of stretch followed by rest, provided in previous 
studies (Björklund et al., 2006; Larsen et al., 2005) did not alter the function of muscle spindles to the same extent as a single continuous stretch.

Several studies have demonstrated that performing moderate intensity warm up exercises improves KJPS ( Bouët \& Gahéry, 2000; Bartlett \& Warren, 2002; Magalhães et al., 2010; Daneshjoo et al., 2012). Moderate intensity exercise increases excitability of intrafusal and extrafusal sensory structures (Bouët \& Gahéry, 2000). However, the significant warm up x stretching type interaction effects present in this study might suggest that both SS and DS provide benefits to KJPS beyond performing moderate intensity exercise alone. However, these additional effects could also be the result of the added volume of exercise resulting from the addition of SS and DS to moderate intensity exercise. Dynamic stretching increases activity of the stretched muscles in a subsequent task (Amiri-Khorasani \& Kellis, 2013), possibly increasing the activity of muscles spindles and subsequently the afferent proprioceptive feedback.

Muscle spindles are the primary detectors of changes in muscle length, and are oriented in parallel with the muscle fibres (Macefield, 2005; Subasi et al., 2008). The findings of this study suggested that stretching the muscle fibres, and subsequently muscle spindles, in conjunction with moderate intensity exercise increases the spindle sensitivity and excitability to a greater extent than performing exercise alone.

Negative effects of SS on muscle strength (Cramer et al., 2004; Power, Behm, Cahill, Carroll, \& Young, 2004) and athletic performance ( Fletcher \& Anness, 2007; Haddad et al., 2014; Trajano et al., 2015) have been established previously, and the findings of the present study are in agreement. Concentric strength of the knee extensors and flexors were greater in both DS and CON compared to SS conditions. Static stretching increases muscle-tendon unit compliance, reducing force transmission from the muscle to skeletal structures, as well as reducing the muscle activation that may be the result of reduced excitability of the motor neuron pool or altered mechanoreceptor excitably (Björklund et al., 2006; Cuissard, Duchateau, \& Hainaut, 1988; Peng et al., 2016; Power et al., 2004; Ye, Beck, \& Wages, 
2016). In contrast, DS demonstrates increased muscle activity following stretching (AmiriKhorasani \& Kellis, 2013), likely due potentiation of the stretched muscle groups caused by the active movement.

Recently DS has been found to improve muscle strength and athletic performance ( Sekir et al., 2010; Haddad et al., 2014; Ryan et al., 2014). The present study found no change in muscle strength following DS and Costa et al. (2014) reported a decrease in hamstring muscle strength following DS. The variable effects of DS reported in previous literature are likely the result of different volumes and intensities of DS (Faigenbaum et al., 2006; Ryan et al., 2014). Costa et al. (2014) performed higher volume DS, comprised of 4 sets of 4 exercises, than the current study, suggesting that high volume DS has similar negative effects on athletic performance as are seen in SS. Further research is needed to determine the interaction between KJPS and muscle strength following different volumes of DS. The use of stretching as part of pre-exercise warm up procedures may reduce the risk of noncontact soft tissue injury. Lower KJPS has also been associated with high risk positions for ACL injury in healthy participants (Mir, Talebian, Naseri, \& Hadian, 2014). The improvement in KJPS following SS and DS is likely due to the stretching procedures altering the function of muscle spindles (Ghaffarinejad et al., 2007), as the sensitivity of muscle spindle intrafusal fibres is increased (Proske, Morgan, \& Gregory, 1993). Proprioception has an important role in stabilising and correctly positioning the knee joint during dynamic exercises (Ogard, 2011). Therefore it is possible that acutely improving KJPS prior to exercise can reduce the likelihood of the knee being placed in high risk positions. However, the decrease in muscle strength following SS could impair athletic performance, limiting its utility in pre-exercise warm-up procedures, despite the potential benefits to knee joint injury risk provided by improved KJPS. In this respect, DS appears to be a suitable compromise. Similar improvements were seen in KJPS between SS and DS, but DS did not result in a decrement to knee extensor and flexor muscle strength, although the previously reported increase in strength (Sekir et al., 2010) was not found using 3 sets of only 1 DS exercise per muscle 
group in the current study. Based on the findings of the present study, it is therefore recommended that if stretching exercises are to be included in pre-exercise warm up procedure DS should be chosen in preference to SS.

In the present study the intensity of stretch for SS and DS was not directly controlled, instead each muscle group was stretched to the participants' volitional maximum range in both SS and DS. Whilst this approach does not specifically control for muscle length and tension it does replicate the approach adopted by previous research (Costa et al., 2014; Fletcher \& Anness, 2007; Ghaffarinejad et al., 2007; Haddad et al., 2014; Larsen et al., 2005; Power et al., 2004; Sekir et al., 2010) to allow for comparison between methods. It is possible that the effect of stretching on muscle strength is dependent on the time the muscle is stretched to the extremity of its range of motion. If this is the case then the longer duration spent at the maximum range of motion in SS than DS may have led to the negative effect on muscle strength after SS, not found for DS. Any effect of stretch duration would also have important implications when comparing between the findings of studies adopting different SS durations or repetitions of DS, and direct comparisons between studies should be made with caution.

The inclusion of both males and females in the study sample may be considered a limitation of the current study. By including both sexes there is a greater possibility for heterogeneity in the response to stretching on KJPS and muscle strength. Furthermore, the control condition used in the present study did not have an equal exercise volume to that of the experimental conditions, by using seated rest in place of the stretching protocols. As such, the effects of stretching on KJPS compared to exercise only may be due to the added volume rather than stretching. Finally, the small sample size used may limit the broader application of these findings, however, the medium and large effect sizes for KJPS and muscle strength variables indicate that the findings of this study have merit beyond the small sample measured.

\subsection{Conclusions}


The findings of this study demonstrate that performing SS or DS of the quadriceps and hamstrings as part of pre-exercise warm up procedures improves KJPS. However, the negative effect of SS on muscle strength will limit its utility as preparation for athletic tasks were maximal force production is a performance component. This study is the first to examine the effect of DS on KJPS and the results suggest that 3 sets of DS for 2 muscle groups about the knee (quadriceps and hamstrings) was sufficient to improve KJPS but did not alter muscle strength. Therefore, DS offers a viable alternative to SS for exercise preparation due to its ability to improve KJPS without detriment to muscle strength.

\section{Conflicts of interest}

No conflicts of interest. This research did not receive any specific grant from funding agencies in the public, commercial, or not-for-profit sectors.

\section{References}

Amiri-Khorasani, M., \& Kellis, E. (2013). Static vs. dynamic acute stretching effect on quadriceps muscle activity during soccer instep kicking. Journal of Human Kinetics, 39, 37-47. https://doi.org/10.2478/hukin-2013-0066

Bartlett, M. J., \& Warren, P. J. (2002). Effect of warming up on knee proprioception before sporting activity. British Journal of Sports Medicine, 36(2), 132-134. https://doi.org/10.1136/bjsm.36.2.132

Bishop, D. (2003). Warm up II: Performance changes following active warm up and how to structure the warm up. Sports Medicine, 33(7), 483-498. https://doi.org/10.2165/00007256-200333070-00002

Björklund, M., Djupsjöbacka, M., \& Crenshaw, A. G. (2006). Acute muscle stretching and shoulder position sense. Journal of Athletic Training, 41(3), 270-274. https://doi.org/10.1016/S0162-0908(08)70082-5

Bouët, V., \& Gahéry, Y. (2000). Muscular exercise improves knee position sense in humans. 
Neuroscience Letters, 289(2), 143-146. https://doi.org/10.1016/S0304-3940(00)012970

Cohen, J. (1988). Statistical power analysis for the behavior sciences (2nd ed.). New York: Erlbaum Associates.

Costa, P. B., Herda, T. J., Herda, A. A., \& Cramer, J. T. (2014). Effects of dynamic stretching on strength, muscle imbalance, and muscle activation. Medicine and Science in Sports and Exercise, 46(3), 586-593. https://doi.org/10.1249/MSS.0000000000000138

Cramer, J. T., Housh, T. J., Johnson, G. O., Miller, J., Coburn, J., \& Beck, T. W. (2004). Acute effects of static stretching on peak torque in women. Journal of Strength and Conditioning Research, 18(2), 236-241.

Cuissard, N., Duchateau, J., \& Hainaut, K. (1988). Muscle stretching and motoneuron excitability. European Journal of Applied Physiology and Occupational Physiology, 58(1-2), 47-52. https://doi.org/10.1007/BF00636602

Daneshjoo, A., Mokhtar, A. H., Rahnama, N., \& Yusof, A. (2012). The Effects of Comprehensive Warm-Up Programs on Proprioception, Static and Dynamic Balance on Male Soccer Players. PLoS ONE, 7(12), 1-10.

https://doi.org/10.1371/journal.pone.0051568

Faigenbaum, A. D., Mcfarland, J. E., Schwerdtman, J. A., Ratamess, N. A., Kang, J., \& Hoffman, J. R. (2006). Dynamic warm-up protocols, with and without a weighted vest, and fitness performance in high school female athletes. Journal of Athletic Training, 41(4), 357-363.

Fletcher, I., \& Anness, R. (2007). The acute effects of combined static and dynamic stretching protocols on fifty-meter sprint performance in trank-and-field athletes. Journal of Strength and Conditioning Research, 21(3), 784-787.

Ghaffarinejad, F., Taghizadeh, S., \& Mohammadi, F. (2007). Effect of static stretching of 
muscles surrounding the knee on knee joint position sense. British Journal of Sports Medicine, 41(10), 684-687. https://doi.org/10.1136/bjsm.2006.032425

Haddad, M., Dridi, A., Chtara, M., Chaouachi, A., Wong, D., Behm, D., \& Chamari, K. (2014). Static stretching can impair explosive performance for at least 24 hours. Journal of Strength and Conditioning Research, 28(1), 140-146.

Larsen, R., Lund, H., Christensen, R., Røgind, H., Danneskiold-Samsøe, B., \& Bliddal, H. (2005). Effect of static stretching of quadriceps and hamstring muscles on knee joint position sense. British Journal of Sports Medicine, 39(1), 43-46. https://doi.org/10.1136/bjsm.2003.011056

Macefield, V. (2005). Physiological characteristics of low-threshold mechanoreceptors in joints, muscle and skin in human subjects. Clinical and Experimental Pharmacology and Physiology, 32, 135-144.

Magalhães, T., Ribeiro, F., Pinheiro, A., \& Oliveira, J. (2010). Warming-up before sporting activity improves knee position sense. Physical Therapy in Sport, 11(3), 86-90. https://doi.org/10.1016/j.ptsp.2010.06.001

Mandelbaum, B. R., Silvers, H. J., Watanabe, D. S., Knarr, J. F., Thomas, S. D., Griffin, L. Y., ... Garrett, Wi. J. (2005). Effectiveness of a Neuromuscular and Proprioceptive Training Program in Preventing the Incidence of Anterior Cruciate Ligament Injuries in Female Athletes : 2-year follow up. The American Journal of Sports Medicine, 33(7), 1003-1010. https://doi.org/10.1177/0363546504272261

Mir, S. M., Talebian, S., Naseri, N., \& Hadian, M.-R. (2014). Assessment of Knee Proprioception in the Anterior Cruciate Ligament Injury Risk Position in Healthy Subjects: A Cross-sectional Study. Journal of Physical Therapy Science, 26(10), 15158. https://doi.org/10.1589/jpts.26.1515

Ogard, W. K. (2011). Proprioception in Sports Medicine and Athletic Conditioning. Strength 
and Conditioning Journal, 33(3), 111-118.

https://doi.org/10.1519/SSC.0b013e31821bf3ae

Peng, Y., He, J., Yao, B., Li, S., Zhou, P., \& Zhang, Y. (2016). Motor unit number estimation based on high-density surface electromyography decomposition. Clinical Neurophysiology, 127, 3059-3065. https://doi.org/10.1016/j.clinph.2016.06.014

Power, K., Behm, D., Cahill, F., Carroll, M., \& Young, W. (2004). An acute bout of static stretching: Effects on force and jumping performance. Medicine and Science in Sports and Exercise, 36(8), 1389-1396.

https://doi.org/10.1249/01.MSS.0000135775.51937.53

Proske, U., Morgan, D. L., \& Gregory, J. E. (1993). Thixotropy in skeletal muscle and in muscle spindles: A review. Progress in Neurobiology, 41(6), 705-721. https://doi.org/https://doi.org/10.1016/0301-0082(93)90032-N

Ryan, E. D., Everett, K. L., Smith, D. B., Pollner, C., Thompson, B. J., Sobolewski, E. J., \& Fiddler, R. E. (2014). Acute effects of different volumes of dynamic stretching on vertical jump performance, flexibility and muscular endurance. Clinical Physiology and Functional Imaging, 34(6), 485-492. https://doi.org/10.1111/cpf.12122

Sekir, U., Arabaci, R., Akova, B., \& Kadagan, S. M. (2010). Acute effects of static and dynamic stretching on leg flexor and extensor isokinetic strength in elite women athletes. Scandinavian Journal of Medicine and Science in Sports, 20(2), 268-281. https://doi.org/10.1111/j.1600-0838.2009.00923.x

Simic, L., Sarabon, N., \& Markovic, G. (2013). Does pre-exercise static stretching inhibit maximal muscular performance? A meta-analytical review. Scandinavian Journal of Medicine and Science in Sports, 23(2), 131-148. https://doi.org/10.1111/j.16000838.2012.01444.x

Subasi, S. S., Gelecek, N., \& Aksakoglu, G. (2008). Effects of different warm-up periods on 
knee proprioception and balance in healthy young individuals. Journal of Sport Rehabilitation, 17(2), 186-205. https://doi.org/18515917

Trajano, G., Pinho, C., Costa, P., \& Oliveira, C. (2015). Static stretching increases muscle fatigue during submaximal sustained isometric contractions. The Journal of Sports Medicine and Physical Fitness, 55(1-2), 43-50.

Ye, X., Beck, T. W., \& Wages, N. P. (2016). Influence of prolonged static stretching on motor unit firing properties. MUSCLE \& NERVE, 53(5), 808-817. https://doi.org/10.1002/mus.24913 
Figures

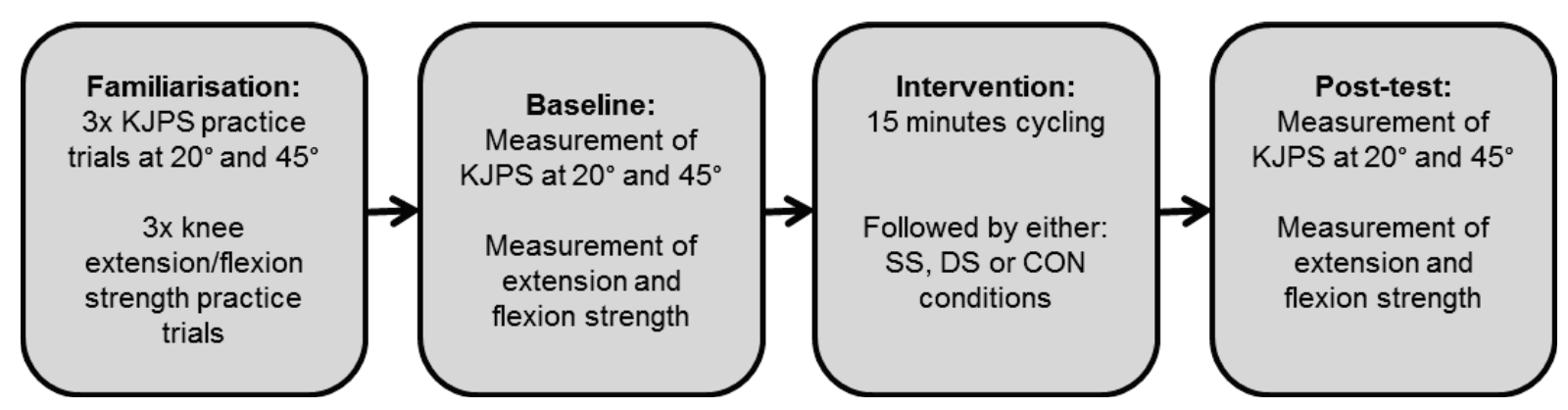

Figure 1. Timeline flow chart for a laboratory visit. Practice trials of knee joint position sense (KJPS) and knee extension and flexion strength tests were followed by baseline KJPS and strength tests. The stretching intervention, either static stretching (SS), dynamic stretching (DS) or no stretching (CON) was followed by post-test KJPS and strength tests.
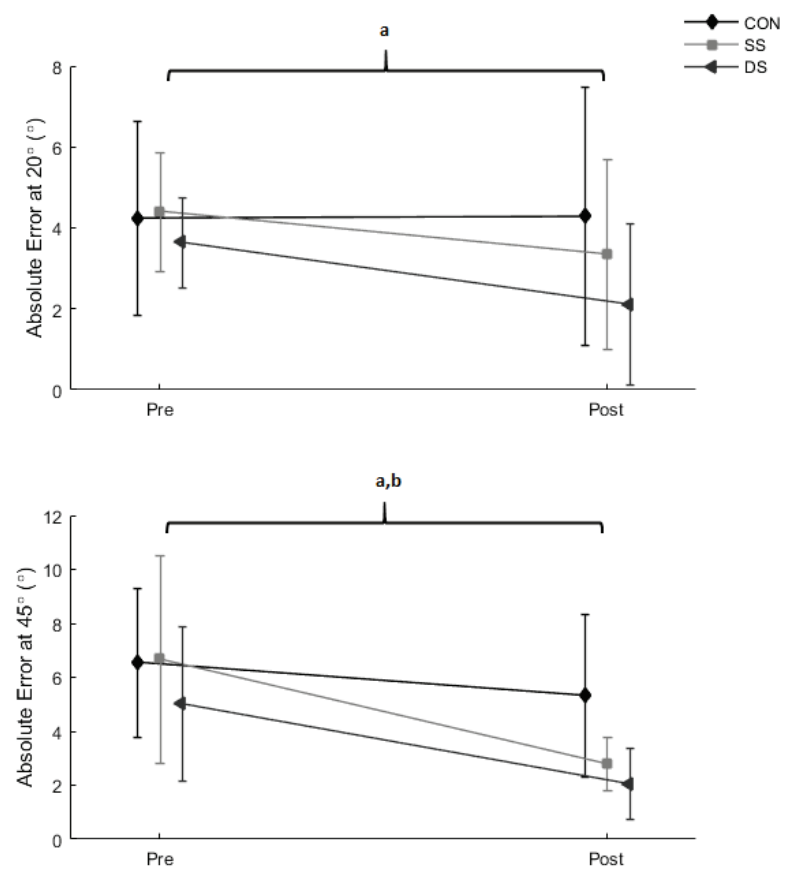

Figure 2. Mean and standard deviations of the knee joint position sense absolute error values for the target angles $20^{\circ}$ (top) and $45^{\circ}$ (bottom) before and after warm up and stretching protocols.

a: significant interaction effect, b: significant warm up effect. 

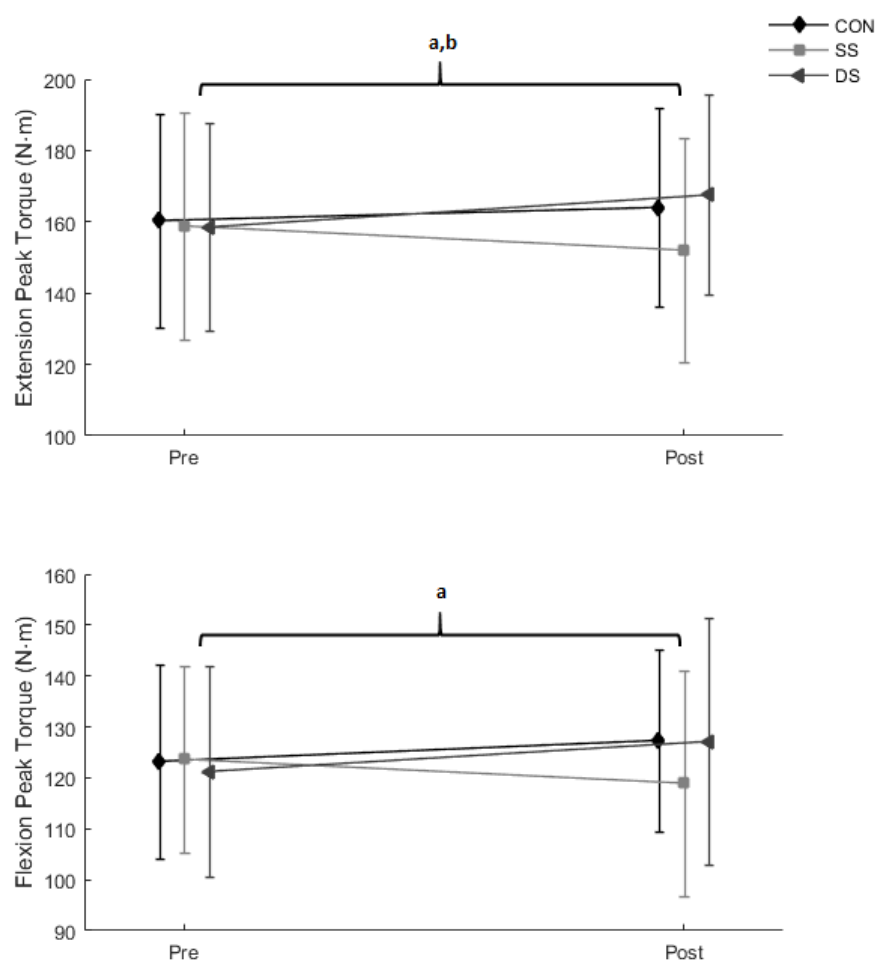

Figure 3. Mean and standard deviations of the knee extensor and flexor concentric peak torque before and after warm up and stretching protocols.

a: significant interaction effect, b: significant stretching type effect. 